\title{
Protecting identity documents by microstructure color differences
}

\author{
Nicolas Rudaz, Roger D. Hersch \\ Ecole Polytechnique Fédérale \\ Lausanne, Switzerland ${ }^{1}$
}

\begin{abstract}
The development of plastic card printers has led to the widespread use of identity documents printed on plastic cards, such as ID cards, driving licenses and access key cards. This paper presents a new security feature based on a technique for embedding a personalized microstructure into an image. This microstructure takes the form of a pattern embedded into the original photograph as a succession of balanced chromatic shifts. The amplitude of these shifts may be tuned so as to make the pattern fully apparent or just noticeable under normal viewing conditions. Since the chromatic shifts cancel each other out in any macroscopic portion of the image, the global appearance of the protected image remains intact. The embedded microstructure may be adapted to each instance of the protected identity document. For example, it can repeat textual information already present elsewhere on the document, or it can include a code derived from data specific to the document holder. Furthermore, this information may be made fully readable without requiring special revealing means. Such identity documents exhibit an intrinsic resistance against imitation, tampering and substitution.
\end{abstract}

Keywords: security printing, identity document, microstructures, color differences

\section{INTRODUCTION}

We are interested in creating color images and security documents with two layers of information, one layer of information being the global image and the second layer of information being represented by a meaningful microstructure embedded within the global image. When seen from far away, only the global image should be visible. From a close viewing distance, the microstructure should provide a clear visual impact.

In the present contribution, we explore a new approach for incrusting human-readable microstructures into color images. An automatic incrustation process synthesizes the final image with the two layers of information. Automatic microstructure incrustation paves the way to the mass production of individually customized security documents.

We incrust microstructures into color images by synthesizing color differences between the foreground and background of the microstructure shapes. When viewed from a large distance, microstructure shapes embedded in the global image should not be distinguishable by the human eye. The resulting perceived color should be a combination of microstructure foreground and background colors, weighted by their respective surfaces. We have much freedom in creating color differences expressed in terms of lightness, saturation or hue difference components. Color differences combining lightness, saturation and hue differences have the potential of increasing the robustness of the document against counterfeiting attempts compared with methods $[1,2]$ that

1.A version of this paper with colorimetrically correct images can be downloaded from the site http://diwww.epfl.ch/w31sp/publications/microstructureimaging/

E-mail: Nicolas.Rudaz@,bluewin.ch, RD.Hersch@epfl.ch

Journal of Electronic Imaging, Vol. 13, No. 2, April 2004, 315 - 323 
modify the lightness and preserve the chromaticity at locations specified by a mask, i.e. within the microstructure foreground.

The first problem consists in creating continuous color differences within a 3D color space. A second problem is the creation of perceptually similar color differences across the color space. A further problem resides in determining the local coverage percentages of the microstructure's foreground and background, in order to appropriately choose their respective colors so as to ensure that when viewed from a certain distance, the combination of foreground and background appears as the desired target color.

\section{CREATING THE MICROSTRUCTURE WITH COLOR DIFFERENCES}

We would like to incorporate the microstructure into pictures by creating color differences between microstructure foreground and background that make the microstructure apparent. However, when viewed from far away, only the global image should remain visible. The microstructure can be made with repetitions of a symbol, a logo, text covering part of the image or ornaments. In the case of a text microstructure, a grid of characters is created and for each character, the respective relative foreground surface coverage is computed (Fig. 9f, color plate). The coverage values within the character's bounding-boxes remain constant. One may smooth out the coverage values at the boundaries between character bounding-boxes by applying a smoothing filter.

To generate an output image incorporating a microstructure, we traverse it pixel by pixel and locate corresponding positions within the original image and within the microtext layer. The color at the current location in the original image and the coverage percentage at the corresponding location of the microstructure (microtext) layer are determined (Fig. 9f). According to the coverage percentage, a color difference pair is formed (see next sections) and depending if the foreground or the background of the microstructure is located at the current location, the one or the other color of the color difference pair is assigned to the current output image pixel.

Fig. 9 illustrates this process with a portrait having the size of the photographs typically used in identity documents printed on plastic cards (Fig. 9a). A bitmap pattern composed of repeated tiles comprising the string "COPYRIGHT / 2001 / EPFL /" is incrusted in the photograph (Fig. 9b). A 300\% magnification of the bitmap pattern (Fig. 9c) and of the final image (Fig. 9d) is provided in order to better appreciate the color differences generated by the incrustation. The text is incrusted into the image with a color difference incorporating a small lightness and strong hue and saturation differences. The lightness and the saturation differences are apparent in the whole image. The hue difference is more subtle, but it can be easily observed under a strong magnification (Fig. 9e). The original photograph and the processed image are nearly indistinguishable when seen from a certain distance.

One may generate color differences along the lightness axis, the saturation axis, the hue axis, or combined differences. A pair of difference colors forms in a linear 3D color space a vector pair having opposite directions. Their respective length ratio is inversely proportional to their corresponding relative surface coverage values (Fig. 10). The color working space must be linear, i.e. the sum of background and foreground colors weighted by their respective relative surface coverage values must generate the resulting target color. When the foreground surface vanishes, i.e. the microstructure disappears, the background color becomes the color of the original image. Color differences along the saturation axis are shown in Fig. 10b and along the hue axis in Fig. 10c. ${ }^{1}$

1. Due to simultaneous contrast, gray surrounded by blue appears as brown. 


\section{SYNTHESIZING COLOR DIFFERENCES}

One would like to exploit the design freedom offered by color differences not only to create lightness variations, but also variations in saturation and hue. For the purpose of reasoning in terms of lightness, saturation and hue color differences, we apply to the RGB color cube a linear transformation comprising a rotation which places the black-white axis in vertical position.

On the planes perpendicular to the black-white axis, saturation and hue are represented in polar coordinates as radius and angle. The resulting coordinate system is named the LEF coordinate system [3]. The LEF lightness (black-white axis) is given by the L coordinate, and the LEF saturation-hue plane is given by the E coordinate (orientation: perpendicular to the $\mathrm{L}$ axis though Red) and the F-coordinate (perpendicular to the $\mathrm{L}$ and $\mathrm{E}$ axes) (see Fig. 1). Other color systems based on lightness (also called value), saturation (also called chroma) and hue such as HLS, HVC, CIELAB [4] are not mathematically linear and therefore cannot be directly used for generating juxtaposed colors whose sum weighted by their respective surface coverages should correspond to a given target color. The YIQ color system [5] is mathematically linear, but it is less intuitive than the proposed LEF system.

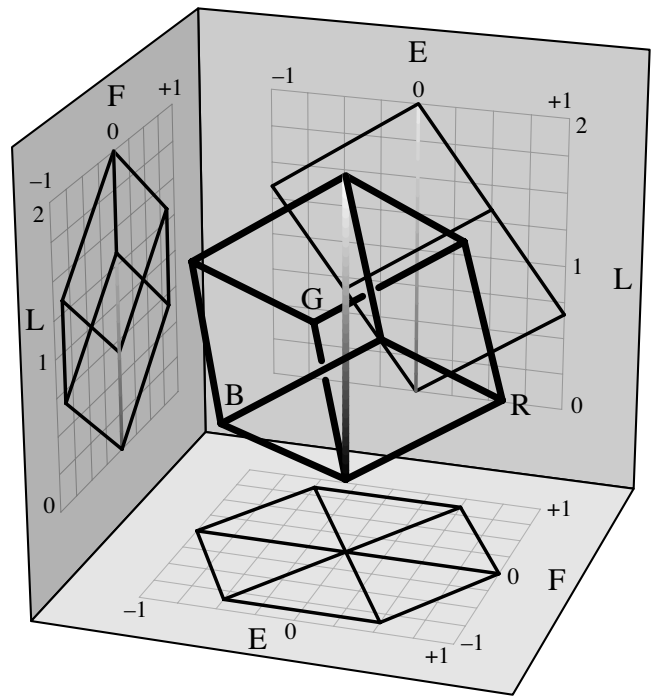

$$
\begin{aligned}
& {\left[\begin{array}{l}
\mathrm{L} \\
\mathrm{E} \\
\mathrm{F}
\end{array}\right]=\left[\begin{array}{ccc}
\frac{2}{3} & \frac{2}{3} & \frac{2}{3} \\
1 & -\frac{1}{2} & -\frac{1}{2} \\
0 & \frac{\sqrt{3}}{2} & -\frac{\sqrt{3}}{2}
\end{array}\right] \cdot\left[\begin{array}{l}
\mathrm{R} \\
\mathrm{G} \\
\mathrm{B}
\end{array}\right]} \\
& {\left[\begin{array}{l}
\mathrm{R} \\
\mathrm{G} \\
\mathrm{B}
\end{array}\right]=\left[\begin{array}{ccc}
\frac{1}{2} & \frac{2}{3} & 0 \\
\frac{1}{2} & -\frac{1}{3} & \frac{1}{\sqrt{3}} \\
\frac{1}{2} & -\frac{1}{3} & -\frac{1}{\sqrt{3}}
\end{array}\right] \cdot\left[\begin{array}{l}
\mathrm{L} \\
\mathrm{F}
\end{array}\right]}
\end{aligned}
$$

Fig. 1 The transformed RGB cube viewed in LEF space and the corresponding linear transformation

Fig. 2 shows constant lightness and constant hue families of slices in the LEF color space. Note that the intersection of a constant lightness slice and a constant hue slice is a radial segment starting at the black-white axis (achromatic color) and ending at the most saturated color located on the volume's external surface.
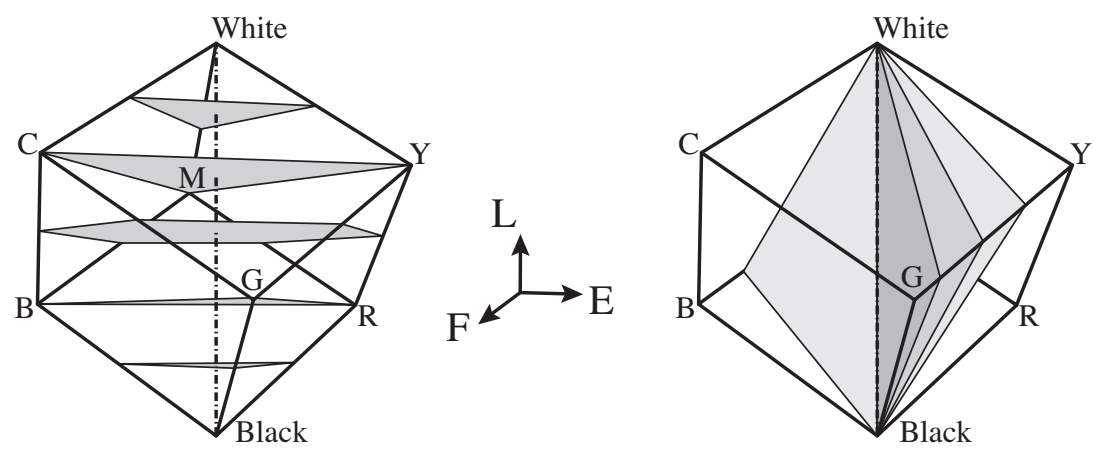

Fig. 2 Constant lightness and constant hue families of slices in LEF color space 
We need a means for creating consistent color differences across the color space according to an initial specification of the amount of LEF lightness, saturation and hue differences. The mapping of an initial color difference onto color differences for colors located within any location of the color space must ensure that for any wedge crossing a part of the color space, color difference vectors are continuous, i.e. no false contours are generated. The principle we follow is to first create for a desired target color a color difference according to the initially specified color difference. In a second step, the created color difference is rectified in order to obtain a color difference that is perceptually similar to all other color differences (see section 4).

In order to obtain a first color difference for the desired target color, the initial color difference is translated parallel to the lightness axis so as to reach the lightness of the target color. It is then rotated around the lightness axis so as to reach the hue of the target color. Finally, it is translated along its radial axis so as to reach the saturation of the target color.

Lightness differences can be created at any point inside the color volume. ${ }^{1}$ However, since one achromatic color can be rendered by an infinite number of color pairs of opposite hues, it makes no sense to define saturation and hue differences on achromatic colors, i.e. on gray tones situated along the black-white axis. Therefore, we require that achromatic colors have only an LEF lightness difference, thus ensuring continuous color differences on a wedge crossing the black-white axis. For colors located between the initially specified rotated color difference and the black-white axis, color differences are obtained by interpolating between the initially specified rotated color difference and a corresponding achromatic lightness difference. ${ }^{2}$ Such an interpolation transforms saturation and hue differences into lightness differences as a function of proximity to the blackwhite axis.

At the boundary or close to the boundaries of the LEF color volume, it is not possible to preserve the initial color difference. We adopt a best effort strategy by modifying when necessary the original saturation difference and keeping as much as possible the hue difference. Fig. 3 shows how saturation and hue differences are adapted in order to remain within the boundaries of the color volume.

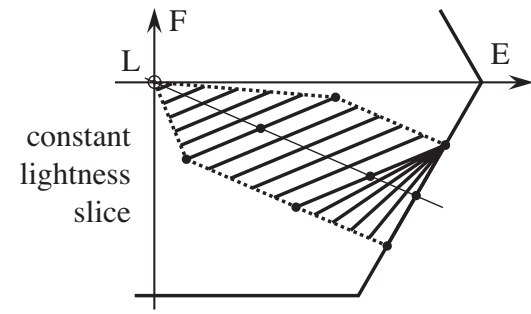

Fig. 3 Adapting saturation and hue differences to remain within the boundaries of the color volume

If necessary, the initial lightness difference is reduced so as to remain within the boundaries of the color volume. As shown in Fig. 4, at positions close to the volume's boundary, we interpolate between the position where the color difference vector touches the volume's boundary and the position where the color difference

1. On points close to the volume boundaries, lightness differences have a range limited by the point's vertical distances to the volume boundaries.

2. The module of the lightness difference can be made equal to the module of the initially specified color difference. 
vector is projected onto the volume boundary and resized. Resizing is carried out in order to avoid an enlargement of the absolute color difference.

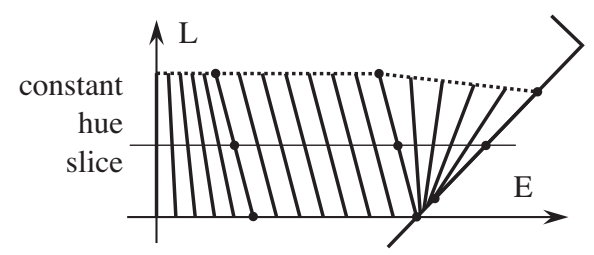

Fig. 4 Adapting LEF lightness and saturation differences at the boundary of the color volume

At the color volume's vertices, all color differences are reduced to zero, i.e. no barycentric combination of colors exists which can render the basic colors black, white, red, green, blue, cyan, magenta, and yellow. Such vanishing color differences are tolerable and may even produce interesting effects. If vanishing color differences need to be avoided, one can slightly desaturate the input image and ensure that it does not incorporate colors located at the color volume's vertices. Then it becomes possible to produce color differences across all image parts.

\section{PERCEPTUALLY SIMILAR COLOR DIFFERENCES}

Our aim is to produce perceptually similar color differences across all or at least most colors of the color volume. It is known that equal color differences (in terms of Euclidean distance) in RGB or CIE-XYZ space do not produce perceptually equal color differences. It is however less known that in the case of large color differences, equal color differences in CIELAB do not produce perceptually equal color differences either [9]. To illustrate this phenomenon, we created in CIELAB a circular color wedge around the lightness axis and generated color differences in lightness, saturation and hue both in the LEF and in the CIELAB color spaces ${ }^{1}$ (Fig. 13).

As can be seen immediately, the perceived lightness differences remain quite similar across the color space both in the LAB and in the LEF spaces. Regarding pure hue and saturation (chroma) differences in both color spaces, the perceived color difference varies as a function of the current color. Perceived color differences in LAB space vary nearly as much as differences in LEF space (printed patches at positions $g$ and $h$ have very close measured $\Delta \mathrm{E}_{\mathrm{Lab}}$ differences of respectively 18.88 and 16.91). This is generally explained by the fact that the CIELAB color system is based on psycho-physical experiments taking into account just noticeable color differences and not large color differences ([7], pp. 306-318).

In certain regions of the LEF space, the perceived color differences become rather low. A more detailed analysis of this phenomenon reveals that this occurs in regions where there is an inversion in the CIELAB lightness differences, i.e. where lightness differences become zero and then grow in the opposite direction. Fig. 5 shows constant lightness curves (fixed L in CIELAB) of a given hue slice in the LEF space. The literature

1. For creating a wedge with CIELAB differences, one needs to transform color coordinates from CIELAB to the display RGB color coordinate system. Conversions from CIE-XYZ to CIELAB and vice-versa are defined in [7]. The conversion from the display RGB values to CIE-XYZ and vice-versa requires a calibrated display with known gamma values and known CIE-XYZ values of the red, green and blue color channels [8]. In our work, images incorporating color differences were generated on a LaCie electron21/108 calibrated display with a gamma value of 1.8 and printed on a dye sublimation Kodak 8670 PS printer using Kodak's color management software. 
mentions that for small color differences (just noticeable differences), lightness differences and chromatic differences seem to be additive [6]. However, when the lightness difference is sufficiently high, it masks the contribution of the chromatic color difference [6]. Therefore, large perceived color differences are produced mainly by CIE-Lab lightness differences. When the CIE-Lab lightness difference becomes zero, hue and saturation differences become apparent, but have a much smaller impact.

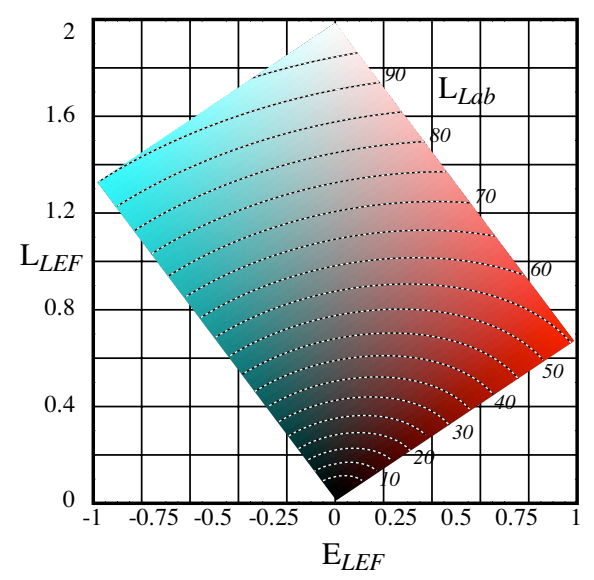

Fig. 5 Constant CIELAB lightness curves within a hue slice in LEF space

A recent study shows that CIELAB lightness differences are consistently judged larger relative to saturation or hue differences. For example, for judging large color differences, some researchers propose to use instead of the CIELAB lightness difference $\Delta \mathrm{L}$ the difference $\Delta \mathrm{L} * 1.54$ ([9], p. 349). Other color systems [10] may be useful for taking into account the color appearance under various observation conditions (illumination, color of surrounding field, etc.) but they do not specifically provide support for large color differences.

In order to create color differences as perceptually similar as possible across the whole color volume, and to avoid CIELAB lightness inversions when producing LEF hue and saturation differences, we limit our study to LEF color differences incorporating a minimal CIELAB lightness difference (i.e. a lightness difference of $\Delta \mathrm{L}_{\mathrm{Lab}} \geq 2$ ).

After having synthesized an LEF color difference according to section 3, a last lightness difference correction step is carried out in order to preserve the originally specified CIELAB lightness difference. For this purpose, we convert the generated LEF difference to CIELAB ${ }^{1}$ and compute by what percentage the LEF difference needs to be reduced or enlarged to fit the CIELAB $\triangle \mathrm{L}$ lightness difference implicitly specified in the initial color difference. Several iterations are needed to converge to the final corrected LEF color difference. These iterations produce a corrected LEF color difference incorporating a CIELAB lightness difference $\Delta \mathrm{L}$ within \pm 0.2 of the initially specified lightness difference.

The importance of this CIELAB lightness correction should not be underestimated when generating photographs with incrusted text. Without correction, the contrast of the incrusted microstructure may vary uncontrollably between too weak and too strong, yielding a text that is illegible in some regions and too contrasted in other regions. Such a contrast variation is illustrated in Fig. 14a, where the incrusted text is legible at position $<1>$, but not at position $<2>$. It is displayed in video inverse mode at position $<3>$. The same photograph

1. LEF colors are converted to CIELAB by converting them first to the display RGB values, then to CIE-XYZ and finally to CIELAB [8, chap. 14] 
processed by applying the CIELAB lightness correction (Fig. 14b) shows a uniformly readable incrusted text that does not interfere with the overall appearance of the picture.

\section{COVERAGE ESTIMATION}

When seen from a certain distance, the foreground color $\mathrm{C}_{\mathrm{f}}$ and background color $\mathrm{C}_{\mathrm{b}}$ of the microstructure contribute to the resulting perceived color $\mathrm{C}$ according to their respective surface coverages $\alpha$ and $(1-\alpha)$ :

$$
\mathrm{C}=\alpha \mathrm{C}_{\mathrm{f}}+(1-\alpha) \mathrm{C}_{\mathrm{b}} ; 0 \leq \alpha \leq 1
$$

Since we would like to compute for a given target color $\mathrm{C}$ the respective colors of foreground $\mathrm{C}_{\mathrm{f}}$ and background $\mathrm{C}_{\mathrm{b}}$, it is important to deduce from the microstructure pattern the relative foreground (or background) coverages within a local neighborhood.

In the case of typographic characters, surface coverages are easily computed by considering the bounding-box of each character and determining the ratio between the discrete character surface (foreground) and the bounding-box surface (Fig. 9f). However, in the case of irregular microstructures of different sizes, the coverages are more difficult to estimate. Depending on the image and on the purpose of microstructure incrustation, we may have various viewing distances. We therefore would like the color difference incrustation to be adaptable to the local periods of the microstructure. The distance between neighboring structures gives some clue about the succession of foreground and background intervals as well as about the bounding-boxes within which the coverage percentages may be computed.

We explore the distance between neighboring structures by starting at the center of the microstructure and launching from a randomly selected initial orientation 16 lines at equally spaced angles, i.e. angles spaced apart by $2 \pi / 32$ (11.25 degrees). For each line (Fig. 6a), we obtain an approximated period $T$ of the microstructure comprising a foreground segment $\tau$ and two half segments of the background color $\left(\mathrm{E}_{1} \mathrm{M}_{1}, \mathrm{E}_{2} \mathrm{M}_{2}\right)$. We also obtain the local $1 \mathrm{D}$ coverage $\mathrm{C}_{\mathrm{L}}=\tau / T$ along that line. The average of the local $1 \mathrm{D}$ coverages along the 16 lines yields the mean local 1D coverage $\alpha_{\mathrm{L}}$ (Fig. 6c). In order to compute a bounding-box corresponding approximately to one structure period, we compute for each main orientation (top, bottom, left, right) the mean location of the period end points (Fig. 6b). The mean local rectangular coverage $\alpha_{R}$ of the microstructure is computed in that bounding box (Fig. 6d).

(a)

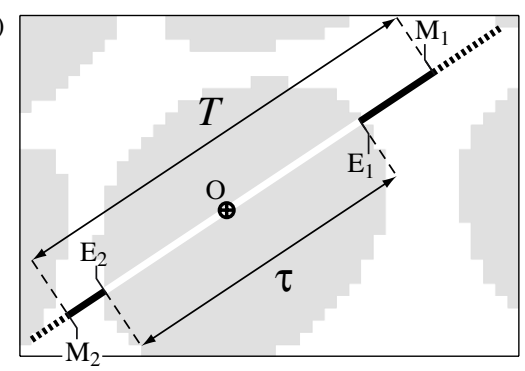

(b)

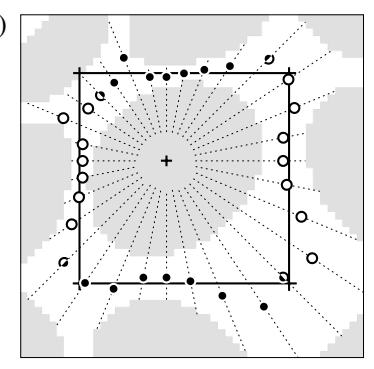

(c)

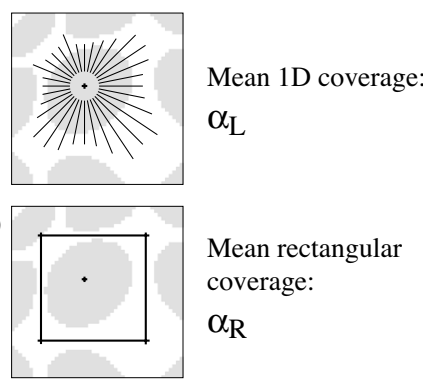

Fig. 6 (a) Approximation of the local microstructure period, (b) determination of its bounding-box by averaging in each main orientation (top, left, bottom, right) the location of the period end points, (c) mean 1D coverage and (d) mean rectangular coverage 
Since some of the lines do not intersect immediate microstructure neighbors, the mean local 1D coverage $\alpha_{L}$ underestimates the microstructure element surface coverage. On the other hand, our experience shows that the mean local rectangular coverage $\alpha_{R}$ overestimates the microstructure element surface coverage. We therefore approximate the final resulting microstructure surface coverage for the analyzed local area as the average of $\alpha_{L}$ and $\alpha_{R}$. When carried out on synthetic examples, the computed microstructure surface coverage is close to the actual surface coverage (within $\pm 10 \%$ ). However, the coverage computation becomes inaccurate when a microstructure is surrounded by a large, empty area.

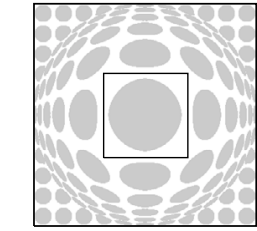

$(1)$

(a)

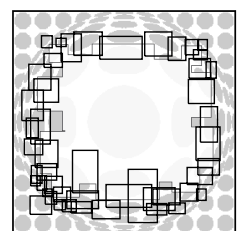

(4)

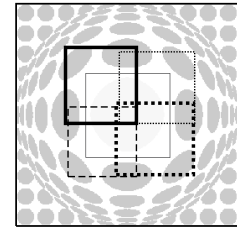

(2)

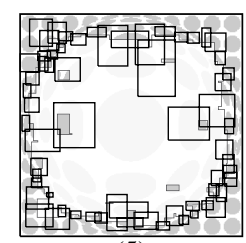

(5)

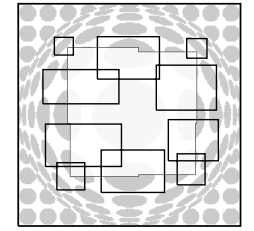

(3)

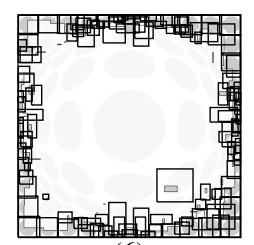

(6)

(b)

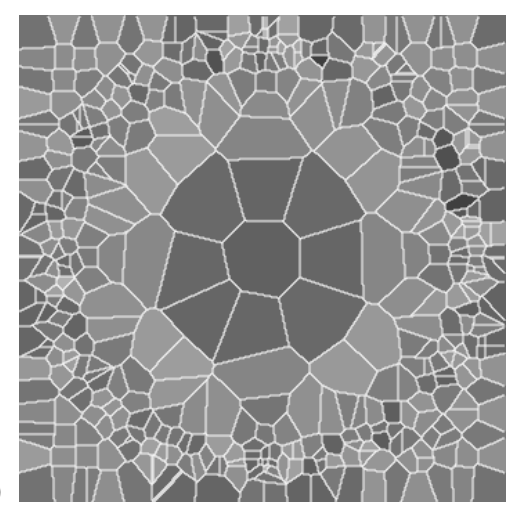

Fig. 7 (a) Computation of coverages in successive regions of the microstructure and (b) Voronoi tesselation of the seed points

The previously computed bounding-box delimits the region for which the coverage is computed. Its vertices and the mid-points between vertices are the new seed points, from which new coverage computations according to the same algorithm are carried out. Seed points located within already computed coverage boundingboxes are removed. Fig. 7a shows the succession of computed regions within the microstructure. At the end of these coverage computation iterations, a local coverage value is associate to each valid seed point. The spatial extent of each seed point is determined by a Voronoi tesselation of the seed points (Fig. 7b). The resulting polygons surrounding the seed points indicate the sub-spaces on which computed local surface coverages remain valid. 
The coverage estimation algorithm described above adapts itself to the local period of the microstructure and yields good results in the general case, i.e. for microstructure elements whose shape and size may vary from instance to instance (Fig. 8).

(a)
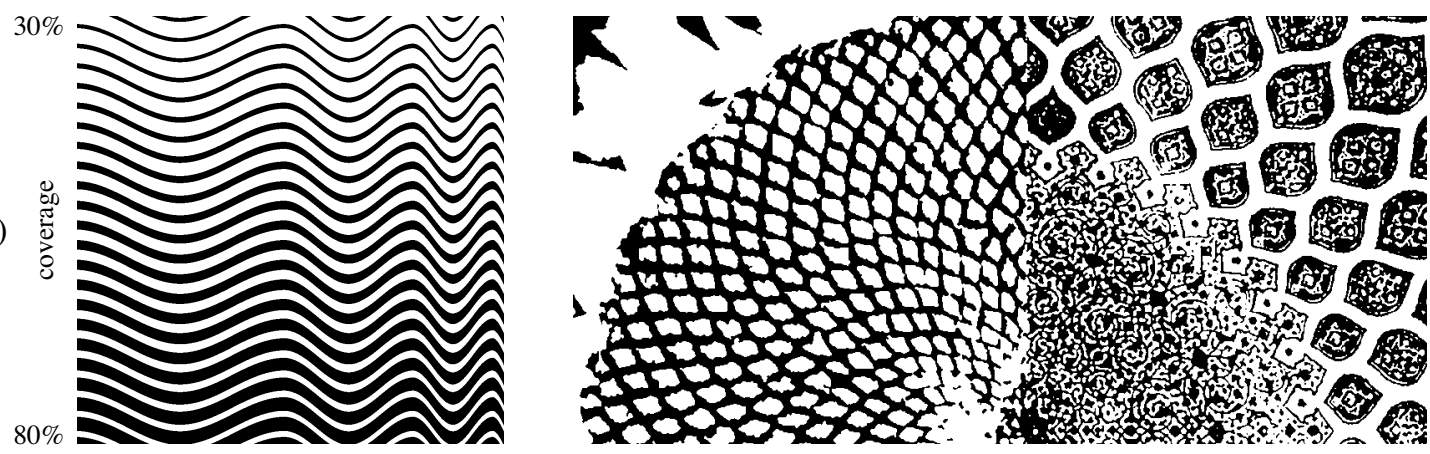

(b)
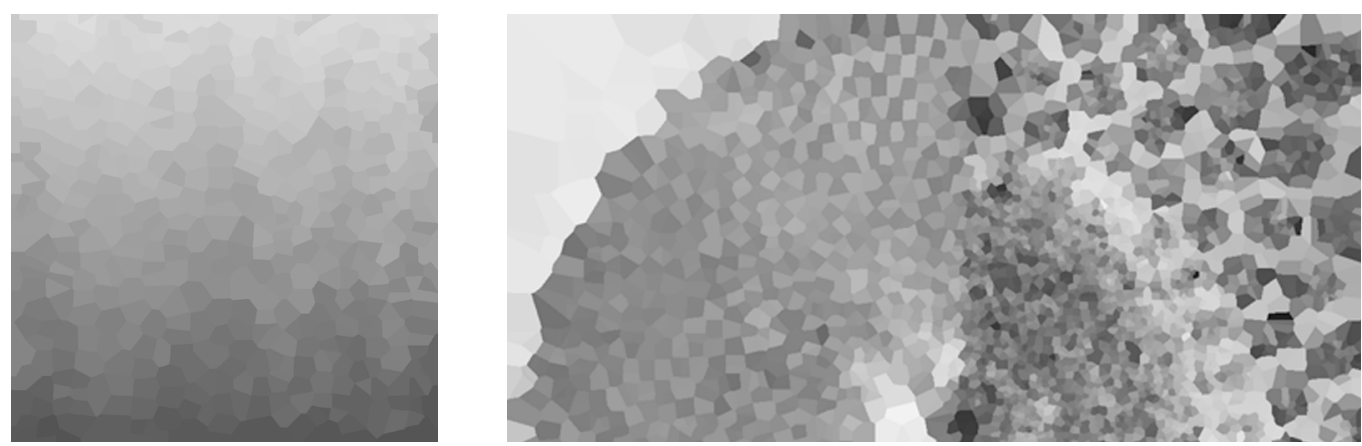

Fig. 8 (a) Microstructures with important coverage and frequency variations, and (b) corresponding computed coverages (darkness proportional to surface coverage)

In order to estimate the quality of the computed coverage map, we consider a synthetic pattern incorporating both different spatial frequencies and abruptly varying coverage values (Fig. 15a). This synthetic microstructure is used for testing the limits of the proposed coverage computation method. When seen from a certain distance, the wedge (Fig. 15b) rendered with a uniform coverage map (Fig. 15c) does not reproduce correctly the original wedge: on the right and left sides of the wedge, horizontal beat patterns are observable (Fig. 15c'). The wedge (Fig. 15d') rendered with the coverage map computed according to the method described above (Fig. 15d) is correctly rendered nearly everywhere. For reference purposes, one can see that the exact, mathematically computed coverage values (Fig. 15e) yield a perfect wedge (Fig. 15e').

\section{SECURITY IMAGES PRODUCED BY COLOR DIFFERENCES}

Incrustation of a microstructure into a color image can also serve as a visible watermark. In contrast to previous visible watermarking techniques [1], [2] which modify the lightness of image parts where the watermark is present, microstructures encrusted with the help of color differences only create local color differences compensating one another. Due to the low pass filter behavior of the human eye, from a certain distance, only the original image color appears.

In the identity card photographs shown in Fig. 9 and Fig. 11, the microstructure encrusted in the images is composed of characters and numbers. A verification code can be incorporated into the text or the numbers encrusted in the image. This text can undergo a geometrical transform, and it is also possible to make it run 
across the whole document in order to further improve its resistance against falsification (Fig. 12). The encrusted color differences are subtle and do not disturb the global face appearance. Under a good light source, one can easily read the underlying text microstructure. The color differences are consistent and look uniform across a broad range of skin colors. Furthermore, the method works also well on black-and-white photographs reproduced as strongly desaturated colors (Fig. 11).

Such identity cards provide both a quick and a thorough visual authentication method. The quick method relies on the verification that the hue of the microstructure foreground actually differs from the hue of the background and that the microstructure foreground color varies for different microstructure color backgrounds. The thorough verification method requires a color chart with for example 125 different color patches, each one with an incrusted microstructure. The inspector must find the color patch closest to the color of a given photograph location and verify that the hue of the microstructure foreground in the photograph actually agrees with the hue of the microstructure foreground in the selected patch. Different variants of our method have been tested in collaboration with company Orell Füssli Security Printing Ltd., and the results we obtained confirmed our expectations. A concrete first application for a driver license was developed and is currently being tested.

On a PowerPC G4 $450 \mathrm{MHz}$ computer, the processing time required for microstructure incrustation into one photograph ( $295 \times 372$ pixels) is inferior to 4 seconds. Incrustation time is proportional to the size of the final image.

\section{CONCLUSIONS}

The challenge resides in creating a global image embedding a microstructure which, when seen from a certain distance, cannot be distinguished from the original image. The microstructure incrustation tool we propose allows to create such images with a minimal amount of human intervention. Designers can concentrate on aesthetic aspects, i.e. choosing a suitable combination of global image and microstructure, as well as a masking layer specifying on which parts of the global image the microstructure is to be applied. After specifying an initial color difference, the incrustation software automatically embeds the microstructure into the global image by synthesizing appropriate color differences.

Generating faithful global images made of repetitive or non-repetitive microstructures requires to solve three problems. The first problem is the synthesis of continuous color differences across the color space. We offer designers the design freedom of specifying color differences in lightness only, in lightness and saturation, in lightness and hue or combining lightness, saturation and hue. The initial color difference is propagated throughout the color volume. At the proximity of the white-black axis, it progressively transforms itself into a pure lightness difference. When it comes close to the volume boundaries, it progressively adapts itself to fit the color volume boundary surface.

The second problem is the generation of perceptually similar color differences across the color volume. In order to avoid inversions of CIELAB lightness differences, we require that the initially specified color difference incorporates a minimal CIELAB lightness difference of $\Delta \mathrm{L}=2$. After computation of a color difference, an iterative correction step is carried out which ensures that the final color difference incorporates whenever possible the initial CIELAB lightness difference.

The third problem is the computation of the local foreground or background coverages for a possibly nonrepetitive microstructure incorporating significant variations in both coverages and local pattern periods. The robust solution we propose consists in specifying a seed point, throwing from that seed point lines at a large number of discrete orientations and analyzing the local pattern period along these lines. The mean values of 
the local pattern periods allow to create a bounding-box surrounding the local pattern. The final local coverage is a function of the line coverages and of the coverage within the bounding-box. Points on the bounding-box are used as new seed points for computing neighboring foreground and background coverages and for propagating the coverage computation iteratively to the full image.

The tool we created allows without much technical efforts to create security documents incorporating freely designed or scanned bi-level microstructures. The incrustation of characters and numbers into valuable document images (e.g. photographs in ID cards) provides a protection against counterfeiting attempts. The incrustation of such information can be specifically adapted to each instance of a security document, increasing its resistance against falsification by substitution.

\section{ACKNOWLEDGMENTS}

We would like to thank Orell Füssli Security Printing Ltd., especially MM. Coleman and Eichenberger for having contributed to this collaborative effort. We also thank the Swiss CTI fund for having partly financed the present research (contract CTI 3776-1).

\section{REFERENCES}

1. US patent 5,530, 759, "Color Correct Digital Watermarking of Images", inventors: W. Braudaway, K.A. Magerlein and F.C. Mintzer, issued June 25, 1996.

2. G. Braudaway, K. Magerlein, and F. Mintzer, "Protecting publicly available images with a visible image watermark", Proc. SPIE: Optical Security and Counterfeit Deterrence Techniques, SPIE-Vol $2659,1996,126-133$.

3. N. Rudaz, R. D. Hersch, V. Ostromoukhov, "Specifying color differences in a linear color space (LEF)", Proc. IS\&T/SID 97 Color Imaging Conference: Color Science, Systems and Applications, Nov. 17-20, 1997, Scottsdale, Arizona, USA, 197-202.

4. J. Foley, A. van Dam, S. Feiner, J.F. Hughes, Computer Graphics. Principles and Practice, 2nd edition, Addison Wesley, 1990.

5. W. K. Pratt, Digital Image Processing, 2nd ed., John Wiley \& Sons, New York, 1991.

6. T.L. Chen, C.Y.Yu, "The Relationship between Visual Acuity and Color Contrast in the OSA Uniform Color Space", Color Research and Applications, Vol 21, No. 1, Feb. 1996, 18-25.

7. G. Wyszecki, W.S. Stiles, Color Science, J. Wiley, 1982.

8. H. R. Kang, Color Technology for Electronic Imaging Devices, SPIE Optical Eng. Press, 1996, Chapter 14.

9. $\quad$ S.S. Guan, M. R. Luo, "A Colour Difference Formula for Assessing Large Colour Differences”, Color Research and Applications, Vol. 24, No 1. 5, Oct. 1999, 344-355.

10. M.D. Fairchild, Color Appearance Models, Addison-Wesley, 1998. 


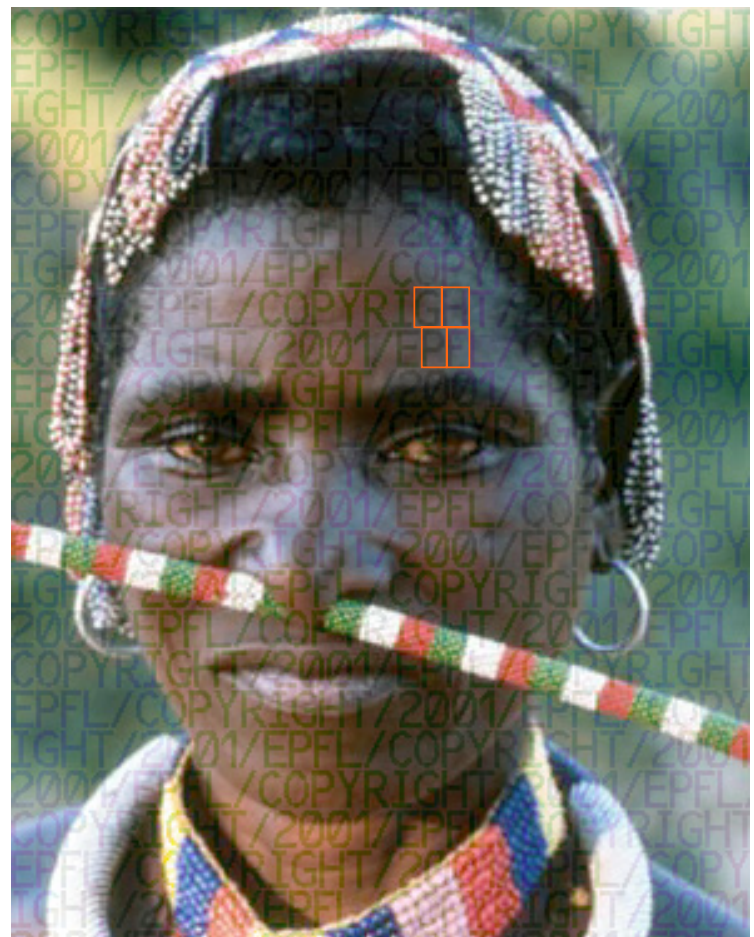

(d)

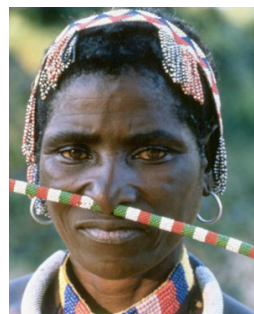

(a)

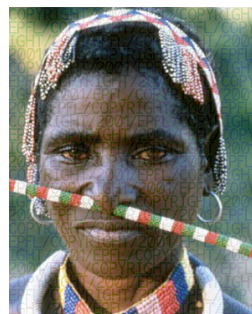

(b)

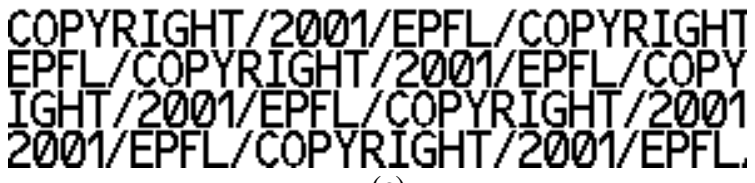

(c)

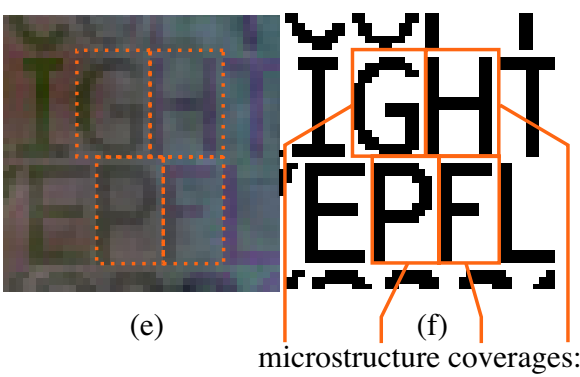

$39 \% \quad 40 \% \quad 35 \% \quad 41 \%$

Fig. 9 Incorporating the microstructure into the image

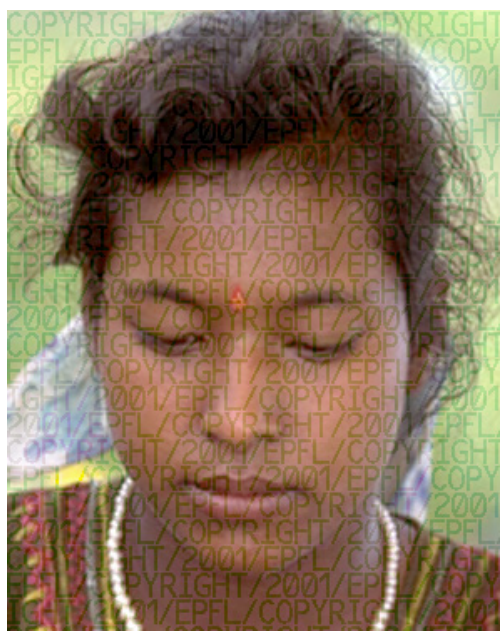

Fig. 10 Saturation differences (b) and hue differences (c) for different surface coverages (a)

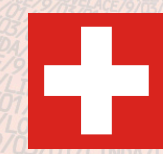

SCHWEIZERISCHE EIDGENOSSENSCHAFT

CONFEDERATION SUISSE

CONFEDERAZIONE SVIZZERA

CONFERDERAZIUN SVIZRA

SWISS CONFEDERATION

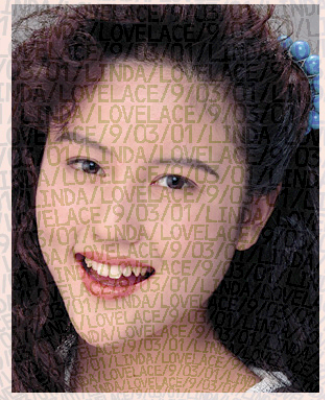

NAME - NOM - COGNOME - NUM - NAME

\section{LOVELACE*}

VORNAME(N) - PRÉNOM(S) - NOME(I) - PRENUM(S) - FIRST NAME(S)

Linda*

GEBOREN AM
NE LE- NATO IL

NASCHI ILS
DATE OF BIRTH

90301

Fig. 12 ID card with geometrically transformed text running over the whole document

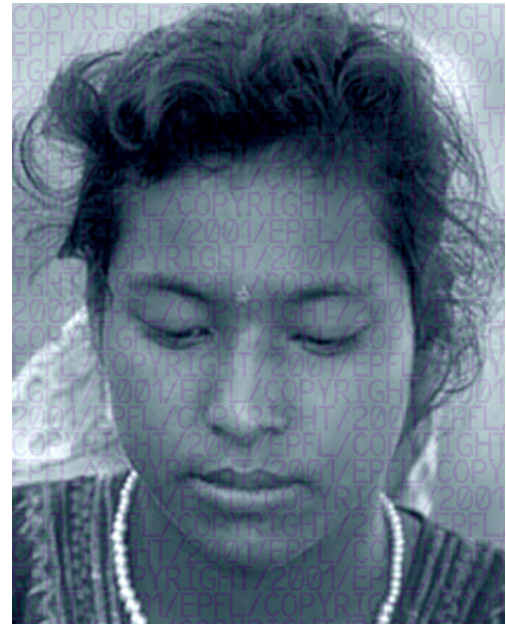

Fig. 11

Encrusted text as a visible watermark in monochrome and color photographs 
Lightness difference of $\Delta \mathrm{E}_{\mathrm{Lab}}=20$

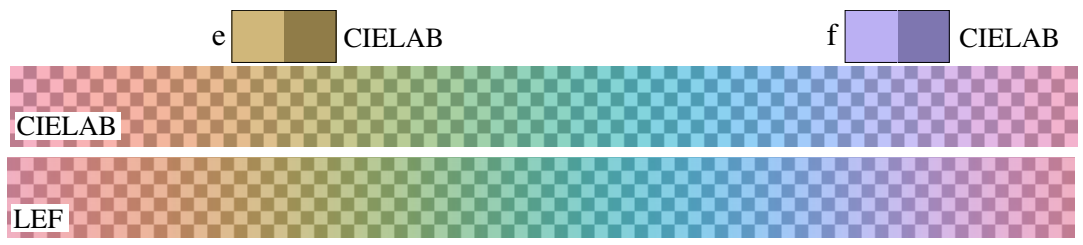

Lightness difference of $\Delta \mathrm{L}_{\mathrm{LEF}}=0.2$

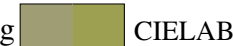

$\mathrm{h}$

CIELAB

Saturation difference of $\Delta \mathrm{E}_{\mathrm{Lab}}=20$

Saturation difference of $\Delta \mathrm{S}_{\mathrm{LEF}}=0.2$

$\begin{array}{ll}\text { CIELAB } & \text { CIELAB } \\ \text { LEF }\end{array}$

Hue difference of $\Delta \mathrm{E}_{\mathrm{Lab}}=20$

$\mathrm{i} \square$ CIELAB $\mathrm{j} \square$ CIELAB

\section{CIELAB}

Hue difference of $\Delta \mathrm{H}_{\mathrm{LEF}}=0.2$

Fig. 13 Circular color wedge around the lightness axis with color differences either in lightness, hue or saturation having equal norms within the CIELAB or within the LEF color spaces

(a)

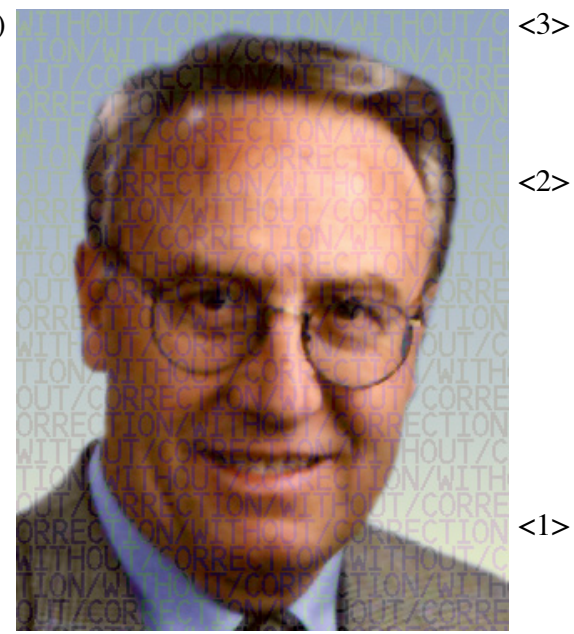

(b)

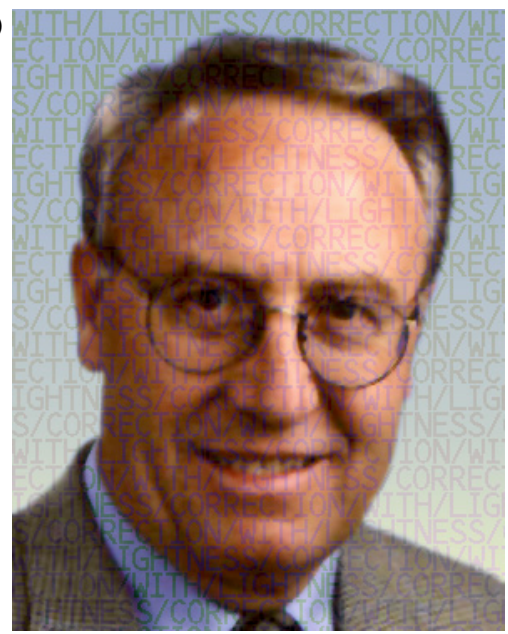

Fig. 14 Incrusted text (a) without and (b) with CIELAB lightness correction

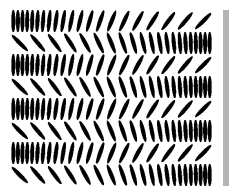

(a)

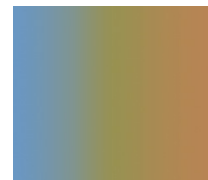

(b)

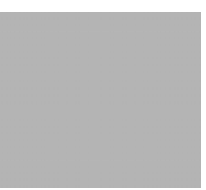

(c)

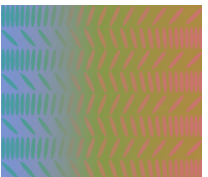

(c')

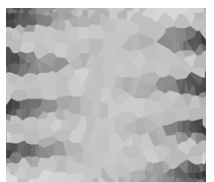

(d)

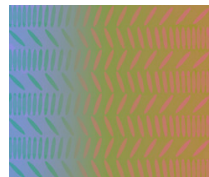

(d')

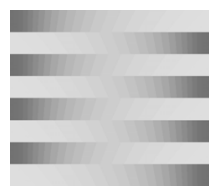

(e)

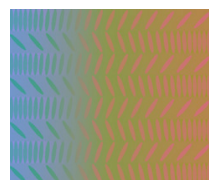

$\left(e^{\prime}\right)$

Fig 15 (a) Synthetic microstructure, (b) original wedge, (c) uniform coverage map, (d) computed coverage map and (e) exact coverage map, as well as the corresponding rendered wedges $\left(c^{\prime}\right),\left(d^{\prime}\right)$ and $\left(e^{\prime}\right)$ 